\title{
CONTRÔLE SPATIO-TEMPOREL DE la lumière en milieux complexes
}

\author{
Mickaël MOUNAIX ${ }^{1,2}$, Hugo DEFIENNE ${ }^{1,3}$, Sylvain GIGAN \\ ${ }^{1}$ Laboratoire Kastler Brossel, ENS-Université PSL, CNRS, Sorbonne Université, Collège de France, 24 rue Lhomond, \\ 75005 Paris, France \\ ${ }^{2}$ School of Information Technology and Electrical Engineering, The University of Queensland, Brisbane QLD \\ 4072, Australia \\ ${ }^{3}$ Department of Electrical Engineering, Princeton University, Princeton, NJ 08544, USA \\ sylvain.gigan@lkb.ens.fr
}

La diffusion de la lumière est souvent considérée comme un obstacle en optique, dont on souhaite s'affranchir. Alors que c'était encore inconcevable au début du siècle (il y a à peine 15 ans !), il est désormais possible de manipuler la lumière cohérente multidiffusée dans des échantillons complexes grâce aux modulateurs spatiaux de lumière. Cette nouvelle thématique, dite du "contrôle du front d'onde ", s'avère très prometteuse pour l'imagerie en profondeur, mais également pour le contrôle de l'interaction lumière-matière en milieu désordonné.

\section{Propagation \\ de la lumière dans les millieux désordominés}

On se représente en général la propagation de la lumière comme un phénomène simple : la lumière se propage en ligne droite, et est déviée sur les interfaces. Une vision « rayon » à grande échelle permet de décrire l'essentiel de la propagation, et la nature ondulatoire de la lumière permet de décrire la résolution, la diffraction, etc. C'est vrai en milieu homogène, comme l'air, le verre, ou un système optique simple comme un télescope ou un microscope. Néanmoins, l'essentiel des milieux qui nous entoure n'est pas homogène : le brouillard, un verre de lait, du papier, et surtout l'essentiel des tissus biologiques sont opaques pour la lumière, c'est-àdire qu'on ne voit pas à travers. Leur comportement optique est lié aux fluctuations spatiales de leur indice de réfraction. On définit alors un libre parcours moyen de diffusion, la longueur moyenne entre deux événements de diffusion. Si la taille à traverser est bien supérieure à cette longueur de diffusion, ces milieux sont dits «multi-diffusants » : la lumière est diffusée de très nombreuses fois avant de les traverser (figure 1). Pour la lumière cohérente, la diffusion multiple donne naissance à des figures d'interférence très complexes : les tavelures, qu'on appelle speckle en anglais. Ces interférences, statiques si le milieu est immobile, sont le signe que la diffusion multiple est un phénomène qui préserve la cohérence, et totalement déterministe.

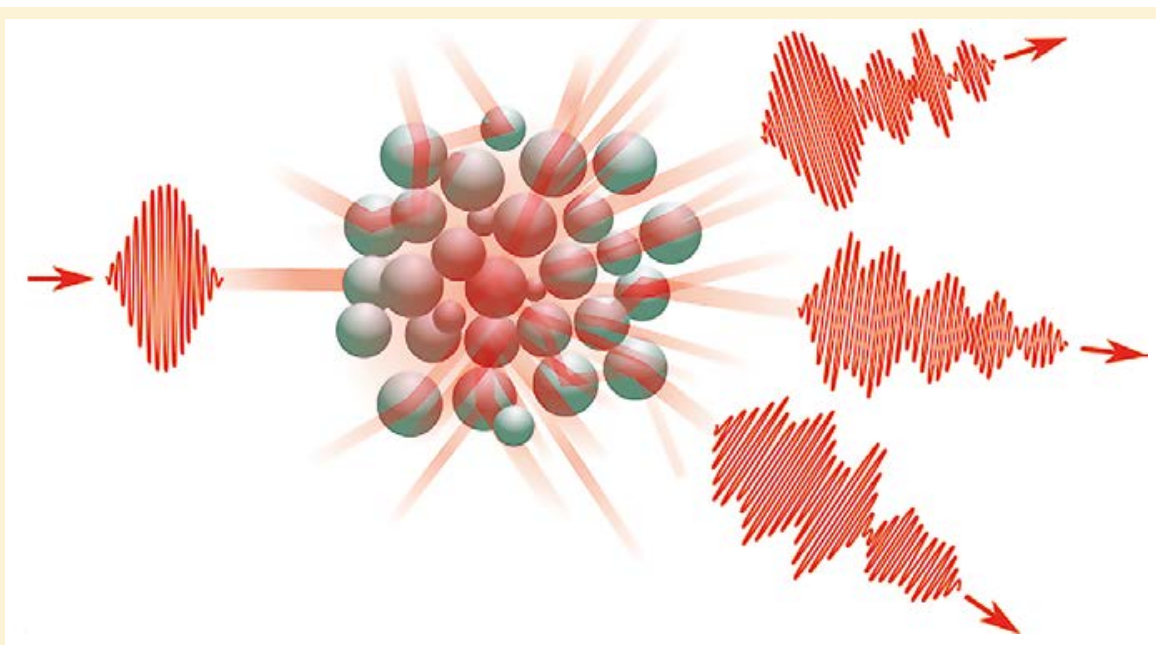

Figure 1. La lumière se propage en diffusant sur les homogénéités du matériau désordonné. L'onde optique détectée en sortie du milieu perd les propriétés de l'onde incidente, comme sa direction, sa forme temporelle ou sa polarisation : c'est un speckle optique. Dans les systèmes sièges de processus de diffusion linéaire, la propagation du champ optique dans le milieu peut se décrire en utilisant un formalisme matriciel. 


\section{Contrôle de la lumière monochromatique à travers les millieux diffusants : le façonnage du front d'onde}

\section{L'outil : le modulateur spatial de lumière (SLM)}

La diffusion de la lumière est un phénomène complexe mais déterministe. Ainsi, la figure de speckle obtenue en sortie du milieu diffusant dépend directement de la manière dont il est illuminé. Cette structure d'illumination se contrôle à l'aide d'un modulateur spatial de lumière (SLM). Un SLM est une matrice de micro-miroirs ou de cristaux liquides (figure 2) qui modulent localement l'amplitude et/ou la phase de l'onde optique qui s'y propage. Les plus couramment utilisés sont les SLMs cristaux liquides à modulation de phase, qui se programment par ordinateur. La technologie des SLMs s'est développée au cours des dernières décennies, notamment grâce à leur utilisation dans les systèmes de vidéo-projection. Contrairement aux miroirs déformables qui étaient utilisés jusqu'alors, les SLMs possèdent une très grande résolution pouvant aller jusqu'à $10^{6}$ pixels par $\mathrm{cm}^{2}$. Par suite, les techniques de contrôle de front d'onde optique se sont développées grâce à la technologie SLM [1]. Elles sont notamment utilisées pour manipuler la propagation de la lumière dans les milieux diffusants.

\section{Focaliser la lumière à travers un milieu diffusant : la lentille opaque}

La première expérience de contrôle de front d'onde optique en milieu diffusant a été réalisée par Vellekoop et Mosk en 2007 [2]. Dans ce travail fondateur, les auteurs modulent l'onde optique qui illumine une couche de peinture afin de re-focaliser la lumière multi-diffusée en sortie du milieu. Le SLM module la phase de l'onde incidente alors que la lumière diffusée transmise à travers le milieu est mesurée avec une caméra. Une boucle de rétrocontrôle est établie entre ces deux éléments et un algorithme d'optimisation itératif maximise l'intensité sur un pixel arbitraire de la caméra. À la fin de l'optimisation, le masque affiché sur le SLM permet aux champs électriques diffusés d'interférer constructivement à la position ciblée, à l'instar d'une lentille convergente. L'image mesurée sur la caméra se compose d'un point brillant émergeant d'un arrière-plan de speckle (figure 2). Le rapport des intensités respectives dépend linéairement du nombre de pixels du SLM utilisé pour effectuer l'optimisation. Ce protocole permet aussi de focaliser la lumière en plusieurs positions simultanément avec un unique masque sur le SLM.

Cette approche d'optimisation permet de contrôler la propagation de la lumière sans connaissances préalables du milieu désordonné. Cependant, elle doit être relancée à chaque changement de cibles de focalisation et ne fournit aucune information sur la structure du milieu désordonné. À la suite de ce travail, une autre méthode de manipulation d'onde plus complète a été développée [3].

\section{Approche matricielle du contrôle du front d'onde}

Dans de nombreux milieux désordonnés, comme une couche de peinture ou un tissu biologique, la composante linéaire des phénomènes de diffusion

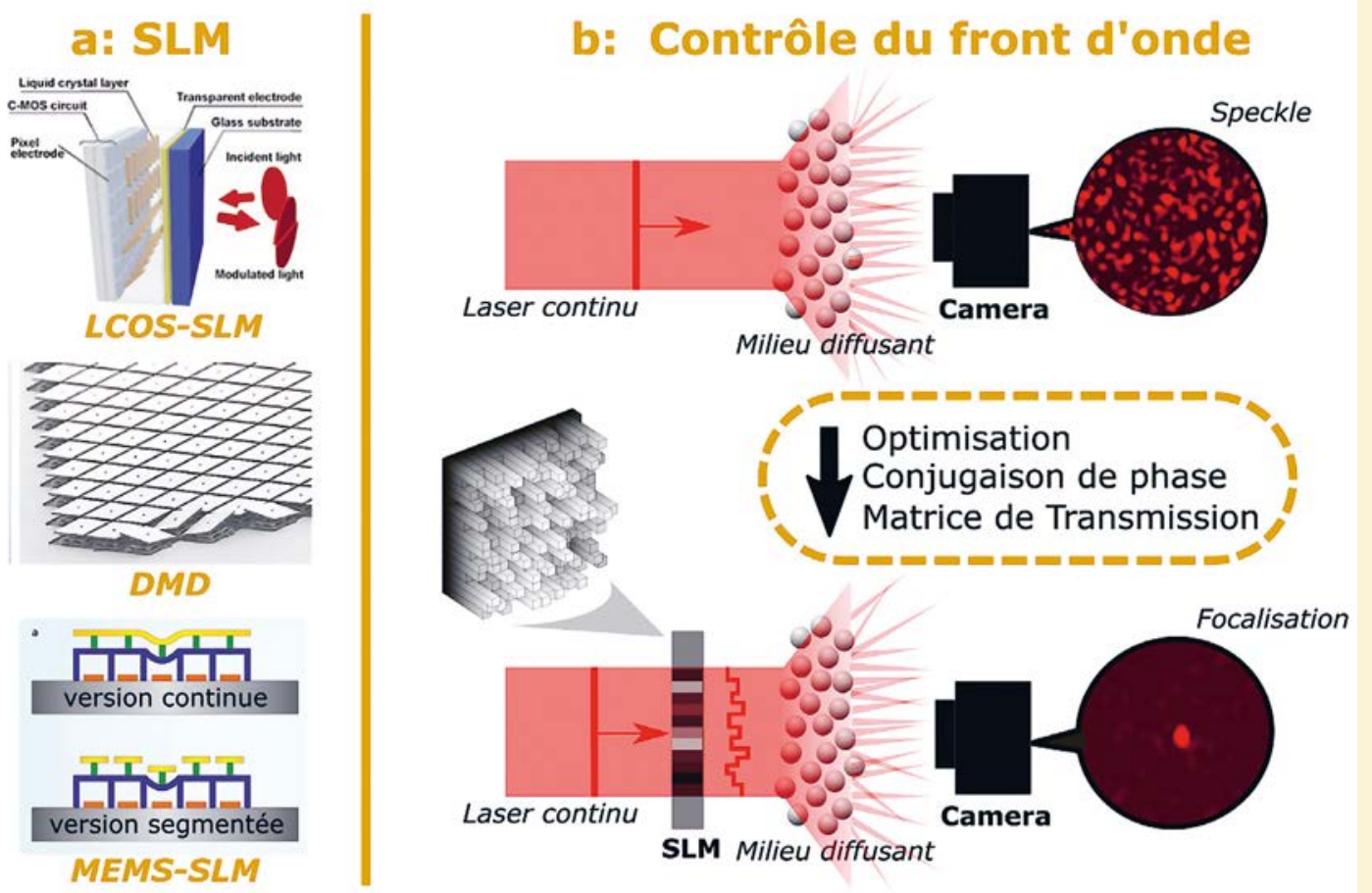

Figure 2. Contrôle du front d'onde de lumière continue se propageant dans un milieu complexe. (a) Différents exemples de SLM. LCOS-SLM : cristaux liquides modulant la phase (Hamamatsu) DMD : micro-miroirs modulant l'amplitude (Texas Instrument) ; MEMS-SLM : micromiroirs modulant la phase (Boston Micromachines). (b)

Focalisation à travers un milieu diffusant. Différentes méthodes permettent de trouver un front d'onde, ajusté par le SLM, pour focaliser la lumière sur une position cible en sortie du milieu. 


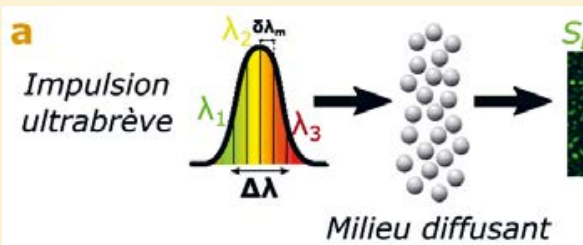

b. Contrôle multi-spectral
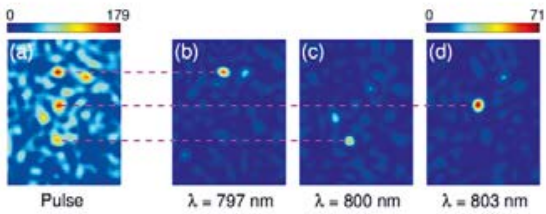

Figure 3. Contrôle spatio-temporel d'une lumière impulsionnelle ultra brève dans un milieu diffusant. (a) Chaque composante spectrale de l'impulsion brève génère son propre speckle en sortie du milieu : on parle de speckle spatio-spectral. (b) Focalisation multispectrale : trois longueurs d'onde sont focalisées sur trois positions spatiales distinctes [7]. (c) Focalisation spatio-temporelle (rouge) de l'impulsion en sortie du milieu comparée à la focalisation de la longueur d'onde centrale (bleue) et au speckle sans contrôle (noir). Les deux signaux ont la même intensité linéaire (L), mais le signal de fluorescence non-linéaire (NL) est plus intense avec l'impulsion re-comprimée temporellement [8].

est dominante : le champ optique diffusé est directement proportionnel au champ incident. L'approche dite «matricielle » du contrôle de la lumière en milieu désordonné exploite directement cette linéarité. Elle s'appuie sur la notion de matrice de diffusion optique. C'est une matrice à coefficients complexes qui relie un champ optique illuminant le milieu à son champ de sortie associé, écrits sous forme vectorielle. Non seulement la matrice de diffusion contient certaines propriétés de désordre du milieu auquel elle est associée, mais elle s'utilise aussi comme un outil pour manipuler la propagation de la lumière en son sein. En pratique, elle se mesure en utilisant un SLM qui module l'onde optique incidente et génère différentes illuminations en entrée [3]. Pour chaque illumination, le champ de speckle correspondant est mesuré en sortie. Il correspond à une colonne de la matrice de diffusion. La matrice du milieu est alors reconstruite colonne par colonne en itérant ce procédé.

Connaître la matrice de diffusion permet de prédire le champ optique de sortie pour n'importe quel champ

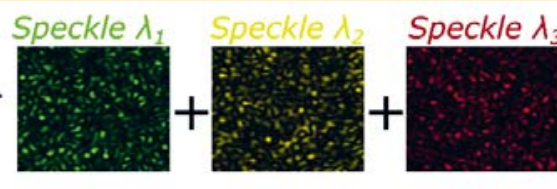

Distorsion spatio-spectrale

\section{Contrôle spatio-temporel}

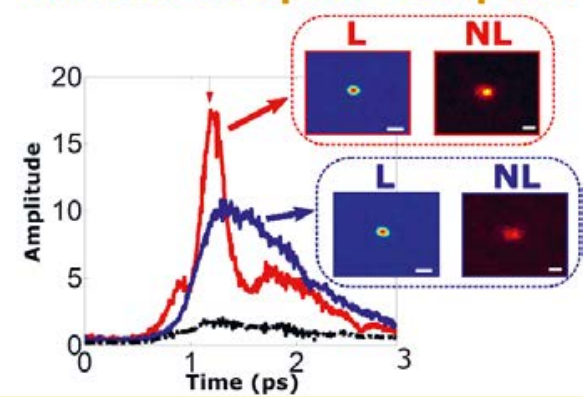

illuminant le milieu. Inverser la matrice de diffusion permet alors de calculer le champ incident associé à un champ de sortie désiré : il est donc possible de programmer le SLM afin de générer un champ optique bien précis à la sortie du milieu, et donc en particulier de focaliser la lumière. Le résultat obtenu est similaire à la focalisation par approche itérative. Cependant, l'étape de mesure de la matrice ne s'effectue qu'une seule fois et cette dernière peut ensuite s'utiliser pour focaliser en n'importe quel point de sortie. D'autre part, la connaissance de la matrice de diffusion permet également de voir à travers un milieu désordonné. En effet, un objet caché derrière un milieu diffusant génère une figure de speckle unique. Connaître la matrice de diffusion du milieu permet de reconstruire l'objet associé à ce speckle particulier [4].

Le formalisme matriciel décrit précédemment ne s'utilise qu'avec de la lumière cohérente monochromatique. Depuis un demi-siècle, les sources lumineuses à large spectre, comme celles générant des impulsions brèves, se sont rendues indispensables pour de nombreuses applications

\section{FORMATION CONTINUE}

\author{
Une nécessité \\ pour les entreprises \\ Une mission majeure
de l'Institut d'Optique
}

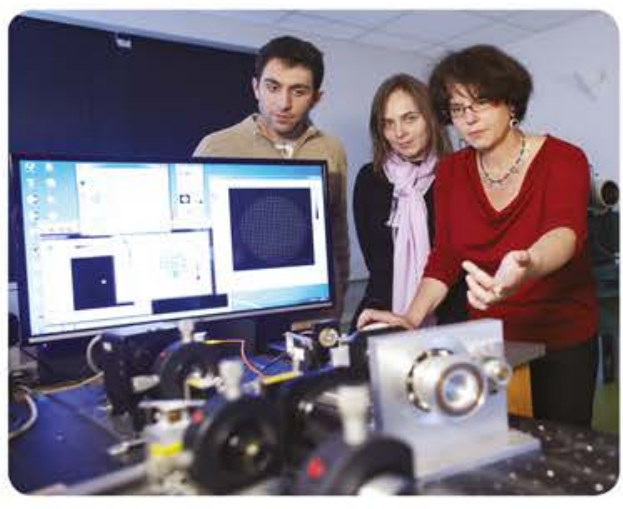

- Au catalogue ou sur-mesure

- Plus de 150 montages pédagogiques

- De l'initiation à la spécialisation

- Formateurs experts

- En anglais ou en français

- En France ou à l'étranger

- Liens forts avec l'industrie

Infrarouge, optronique

- Instrumentation, composants,

fabrication

- Conception optique

- Imagerie, mesure, communication

- Sources, éclairage, lasers, sécurité

- Mastère Spécialisé ${ }^{\circledast}$ ELS

Systèmes d'Éclairage Embarqué

Nous contacter

Emilie Ericher

+33164533236

fc@institutoptique.fr 

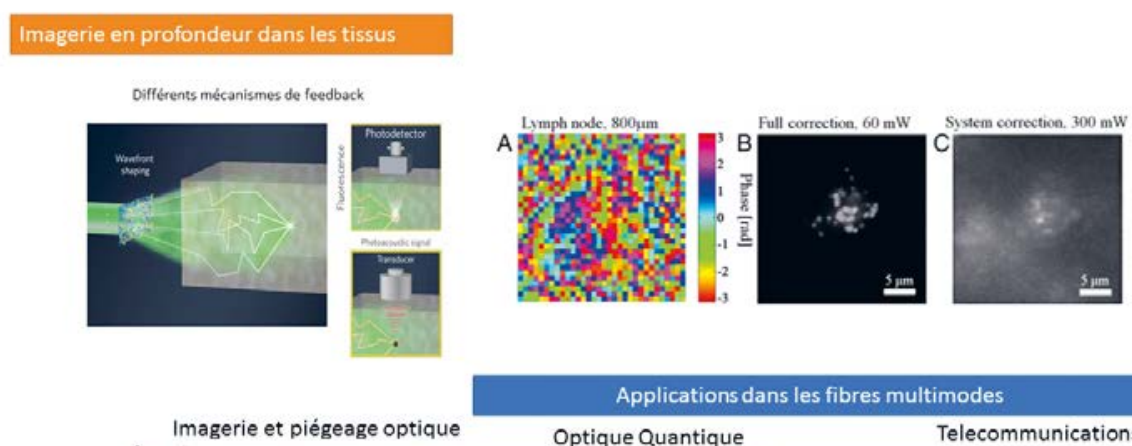

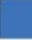
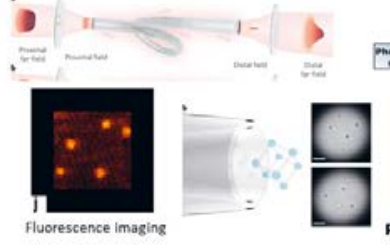
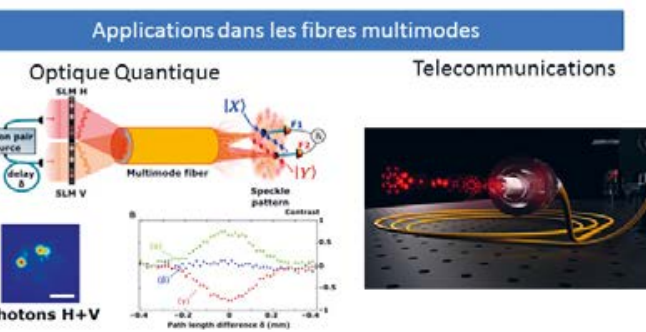

Telecommunications

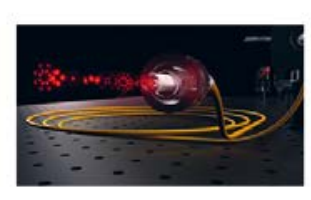

Optique Quantique
Figure 4. Quelques applications du contrôle de front d'onde en milieux complexes. (Haut) Imagerie en milieu biologique. Le challenge est d'avoir un moyen de focaliser à l'intérieur du milieu. Différentes approches, basées sur l'acoustique ou la fluorescence, ont été proposées, mais reste un problème ouvert [9]. Néanmoins, de premières démonstrations en imagerie biologique ont été réalisées [10]. (Bas) Un domaine d'application particulièrement intéressant sont les fibres multimodes, où ces techniques de contrôle de front d'onde ont trouvé de nombreuses applications en imagerie, pinces optiques [11], mais aussi en optique quantique [12] et télécommunications. (Crédit : Joel Carpenter, The University of Queensland, Australie) d’optique non-linéaire. En généralisant ce formalisme aux sources à large spectre, il devient possible d'utiliser des approches d'optique non-linéaire en milieu désordonné, en particulier pour développer des techniques d'imagerie biologique en profondeur.

\section{Contrôle de la lumière à large spectre à travers les milieux diffusants}

\section{Propagation d'une impulsion brève dans un milieu complexe}

Les sources impulsionnelles ultra-brèves génèrent des impulsions optiques courtes temporellement, avec des durées de l'ordre de 100 fs pour un oscillateur titane:saphir. Réciproquement, elles sont larges spectralement, avec une bande spectrale de l'ordre de $10 \mathrm{~nm}$. Pour un profil d'amplitude donné, la durée d'une impulsion est la plus brève possible si sa phase spectrale associée est constante : l'impulsion est dite limitée par transformée de Fourier. Des lors que celle-ci n'est plus plate, l'impulsion s'allonge et sa puissance crête, à laquelle sont sensibles les effets d'optique non-linéaire, diminue.

Lorsqu'une impulsion brève se propage dans un milieu désordonné, la lumière subit de nombreux phénomènes de diffusion successifs et parcourt des chemins optiques de différentes longueurs. Similairement au cas monochromatique, les ondes diffusées se combinent et interferent en sortie du milieu pour former une figure de speckle spatio-temporel : l'onde diffusée est désordonnée spatialement (speckle spatial) mais aussi temporellement (speckle temporel). De manière équivalente, le désordre temporel observé en sortie s'interprète comme la superposition incohérente de speckles spatiaux associés aux différentes composantes spectrales de l'impulsion (figure 3). L'amplitude et la phase spectrale de l'onde mesurée à une position spatiale donnés sont distordues, générant une impulsion temporelle désordonnée et rallongée avec une puissance crête amoindrie. La diffusion limite ainsi la capacité des techniques d'imagerie non-linéaire dans les milieux désordonnés.

\section{Focalisation spatio-temporelle d'une impulsion brève à travers un milieu complexe}

Afin de manipuler un speckle spatio-temporel, il faut contrôler les propriétés spectrales de l'onde diffusée, en plus de ses propriétés spatiales. De manière surprenante, ce contrôle peut se réaliser en utilisant un unique SLM. En effet, à l'instar d'un réseau de diffraction, le milieu diffusant couple les propriétés spatiales et spectrales de la lumière, mais de manière complexe.

Dans les premiers travaux de focalisation spatiale et temporelle d'une impulsion brève diffusée, une approche itérative a été utilisée. Le signal de rétro-contrôle peut être linéaire, comme l'intensité de l'impulsion mesurée en sortie à un temps bien précis [5], ou non-linéaire comme un signal de fluorescence [6]. Récemment, nous avons étendu l'approche matricielle, décrite précédemment pour le cas monochromatique, au cas de la propagation d'impulsions ultra-brèves (donc très multichromatiques). En pratique, la matrice de diffusion spatiospectrale se reconstruit en mesurant un ensemble de matrices de diffusion monochromatiques pour chaque longueur d'onde qui composent la bande spectrale de l'impulsion brève.

La matrice spatio-spectrale du milieu diffusant capture la complexité du processus de diffusion, et notamment les propriétés du couplage spatio-spectral. Elle peut par exemple s'utiliser pour transformer le milieu diffusant en un réseau de diffraction contrôlable : différentes longueurs d'onde sont focalisées à différentes positions spatiales en sortie du milieu (figure 3) [7]. 
Focaliser toutes les longueurs d'onde à la même position spatiale avec une phase constante permet une compression de l'impulsion de sortie à sa limite de transformée de Fourier : l'impulsion est alors focalisée spatialement et temporellement, sans utiliser d'approche itérative (figure 3). D'autre part, la richesse de l'information contenue dans la matrice permet une manipulation très précise du champ de sortie. Par exemple, le temps d'arrivée de l'impulsion est accordable, tout comme la génération d'impulsions doubles, utiles dans des expériences de type «pompe-sonde» [8]. Le SLM et la matrice de diffusion spatio-spectrale permettent donc de compenser la dispersion spatiale et temporelle de l'onde diffusée à travers le milieu, afin par exemple d'exalter un signal de fluorescence d'une cible située de l'autre côté du milieu (figure 3).

\section{Les futuiss défis}

Le contrôle cohérent de la lumière en milieu complexe, tant spatial que temporel, est donc un concept qui a déjà révolutionné notre vision de la diffusion multiple. Ce n'est plus seulement une perturbation à éviter à tout prix, mais elle peut être compensée, voire mise à profit. De plus, de nouvelles fonctions et applications, inimaginables il y a encore 10 ans, sont maintenant envisageables (figure 4) : imager à grande profondeur dans les tissus biologiques, transmettre plus de données dans une fibre multimode, des endoscopes plus fins, des spectromètres ultracompacts, etc. Les challenges technologiques sont nombreux, et de nombreuses questions fondamentales, liées à la physique de la propagation des ondes en milieux complexes, restent à explorer.

\section{POUR EN SAVOIR PLUS}

[1] Stefan Rotter, Sylvain Gigan, Light fields in complex media: Mesoscopic scattering meets wave control, Rev. Mod. Phys. 89, 015005 (2017)

[2] I.M. Vellekoop, A.P. Mosk, Focusing coherent light through opaque strongly scattering media, Opt. Lett. 32, 2309-2311 (2007)

[3] S.M. Popoff, G. Lerosey, R. Carminati, M. Fink, A.C. Boccara, S. Gigan, Measuring the transmission matrix in optics: an approach to the study and control of light propagation in disordered media, Phys. Rev. Lett. 104, 100601 (2010)

[4] Sébastien Popoff, Geoffroy Lerosey, Mathias Fink, Albert Claude Boccara, Sylvain Gigan, Image transmission through an opaque material, Nat. Commun. 1, 81 (2010)

[5] Jochen Aulbach, Bergin Gjonaj, Patrick M. Johnson, Allard P. Mosk, Ad Lagendijk, Control of light transmission through opaque scattering media in space and time, Phys. Rev. Lett. 106, 103901 (2011)

[6] Ori Katz, Eran Small, Yaron Bromberg, Yaron Silberberg, Focusing and compression of ultrashort pulses through scattering media, Nature Photonics 5, 372 (2011)

[7] Daria Andreoli, Giorgio Volpe, Sébastien Popoff, Ori Katz, Samuel Grésillon, Sylvain Gigan, Deterministic control of broadband light through a multiply scattering medium via the multispectral transmission matrix, Sci. Rep. 5, 10347 (2015)

[8] Mickael Mounaix, Daria Andreoli, Hugo Defienne, Giorgio Volpe, Ori Katz, Samuel Grésillon, Sylvain Gigan, Spatiotemporal coherent control of light through a multiple scattering medium with the multispectral transmission matrix, Phys. Rev. Lett. 116, 253901 (2016)

[9] Roarke Horstmeyer, Haowen Ruan, Changhuei Yang, Guidestar-assisted wavefront-shaping methods for focusing light into biological tissue, Nature Photonics 9, 563 (2015)

[10] Jianyong Tang, Ronald N. Germain, Meng Cui, Superpenetration optical microscopy by iterative multiphoton adaptive compensation technique, PNAS 109, 8434 (2012)

[11] Ivo T. Leite, Sergey Turtaev, Xin Jiang, Martin Šiler, Alfred Cuschieri, Philip St. J. Russell, Tomáš Čižmár, Three-dimensional holographic optical manipulation through a high-numerical-aperture soft-glass multimode fibre, Nature Photonics 12, 33 (2018)

[12] Hugo Defienne, Marco Barbieri, Ian A. Walmsley, Brian J. Smith, Sylvain Gigan, Two-photon quantum walk in a multimode fiber, Science Advances 2, e1501054 (2016)

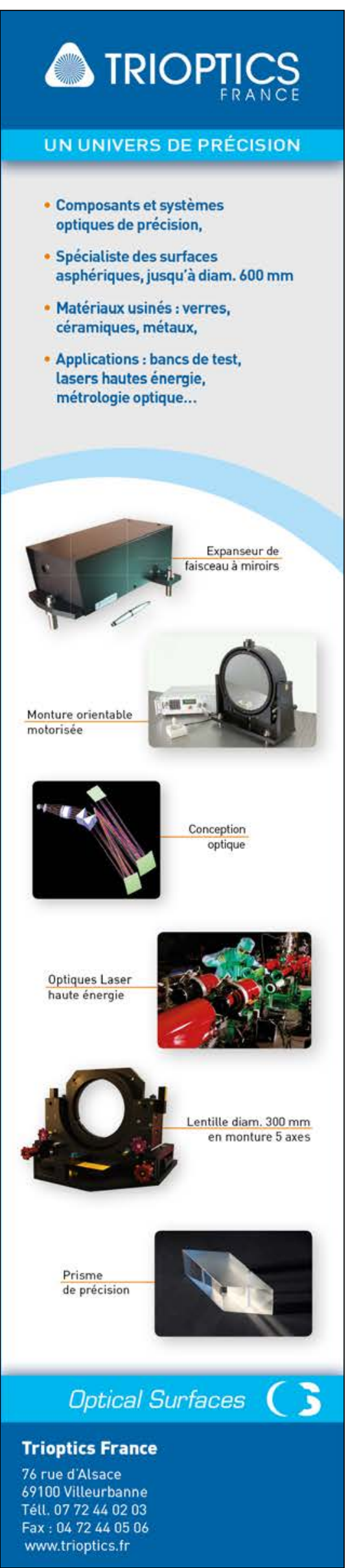

